\section{Commentary: When a cardiac surgeon takes care of patients with Coronavirus Disease 2019: It's gonna be ok!}

\author{
Antonio Miceli, MD, PhD
}

Pandemic, lockdown, stay home. Words that nobody would have ever wanted to hear. An invisible enemy, widespread everywhere, that causes fear, agony, and deaths. It has been recorded that more than 1.5 million people have been affected by Coronavirus Disease 2019 (COVID-19) and 85,000 have died. ${ }^{1}$ Unfortunately, data will surge. After China, Italy was struck first, requiring a reorganization of the health system. In Lombardy (the most hit Italian region), hospitals saturated their bed availability, overwhelmed with patients with COVID-19. Intensive care units crashed, requiring the transfer of patients to other hospitals outside of the region or in some cases outside of the country. Since the beginning of March, all elective procedures stopped, guaranteeing only urgent or emergency operations. The regional health system identified 4 hub centers of 20 cardiothoracic departments in Lombardy, serving more than 10 million people. Despite a large amount of patients, few patients have undergone operations per week, pointing out that people fear COVID-19 rather than a heart attack or dissection.

My lifestyle has changed and my job has changed since I started taking care of patients with COVID-19. Nevertheless, a cardiothoracic hub center allowed my team to take care of 1 urgent case from our waiting list. Per policy, the following protection procedures were established:

1. Self-isolation for at least 14 days (incubation period) whenever possible.

\footnotetext{
From the Minimally Invasive Cardiothoracic Department, Istituto Clinico Sant'Ambrogio; and COVID-19 Department, Istituto Clinico Sant'Ambrogio, Milano, Italy. Disclosures: The authors reported no conflicts of interest.

The Journal policy requires editors and reviewers to disclose conflicts of interest and to decline handling or reviewing manuscripts for which they may have a conflict of interest. The editors and reviewers of this article have no conflicts of interest.

Received for publication April 8, 2020; revisions received April 8, 2020; accepted for publication April 10, 2020; available ahead of print April 15, 2020

Address for reprints: Antonio Miceli, MD, PhD, Istituto Clinico Sant'Ambrogio, Minimally Invasive Cardiac Surgery Department, Via LG Faravelli 16, Milano, Italy (E-mail: antoniomiceli79@alice.it).

J Thorac Cardiovasc Surg 2020;160:e43-4

$0022-5223 / \$ 36.00$

Copyright (c) 2020 by The American Association for Thoracic Surgery

https://doi.org/10.1016/j.jtcvs.2020.04.027
}

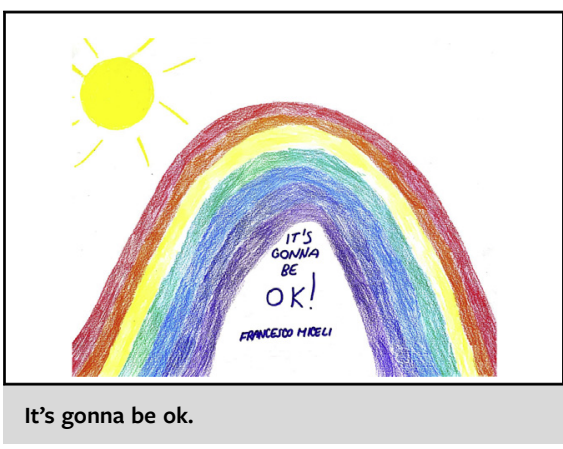

\begin{abstract}
CENTRAL MESSAGE
The strict observance of protective measures and the interruption of elective surgical procedures enhance the security and safety of patients and health care personnel.
\end{abstract}

2. Nasal swab screening for COVID-19 done the same day of admission in hospital, keeping the patient isolated as with COVID-19 infection.

3. All medical and nurse staff must wear personal protective equipment until a swab test shows negative results.

4. All surgical staff has an FFP3 mask plus a surgical mask during operations to avoid contamination from patient to surgeon and vice versa.

5. Intensive care, high dependency unit, and ward COVID19 free.

Despite these measures, there is still a percentage of COVID-19 infection. Data from other hospitals show there are still some patients infected who may not receive this strict surveillance. The risk of not following the policy of personal protective equipment by health personnel, asymptomatic patient visitors, and false-negative and precocious swab tests may cause COVID-19 spread in hospitals.

To reduce the risk of severe acute respiratory syndrome coronavirus 2 nosocomial transmission, Mayeur and colleagues ${ }^{2}$ propose suspending all preoperative nasal swab screening for Staphylococcus aureus. ${ }^{2}$ Patients undergoing cardiac surgery who have $S$ aureus also have a greater risk of surgical site infection, and their identification is mandatory for good outcome. ${ }^{3}$ I agree with the authors that all patients should be considered positive for $S$ aureus. However, in this world crisis, the focus is COVID-19 infection. All patients with cardiac disease should be considered positive for COVID-19, and elective cardiac surgery has to be stopped. 
The risk of dying of pneumonia is too high for low-risk surgery. It seems that the average person infected with coronavirus contaminates 2 to 3 people. In light of my considerations, only emergency and urgent operations should be performed. The strict observance of protective measures and the interruption of elective surgical procedure will enhance the security and safety of patients and health care personnel. While the infection advances in other countries, the social distancing has brought its first positive effects in Italy. In the meantime, we learned the importance of a hug with friends and family, solidarity, and time. We will win this war and see the rainbow. It's gonna be ok!

\section{References}

1. COVID 19 coronavirus pandemic; . Available at: https://www.worldometers.info/ coronavirus/. Accessed April 8, 2020.

2. Mayeur N, Berthoumieu P, Charbonneau H. Does nasal screening for Staphylococcus aureus before surgery compromise health care professional safety in COVID-19 era. J Thorac Cardiovasc Surg. 2020;160:e39.

3. Hong JC, Saraswat MK, Ellison TA, Magruder JT, Crawford T, Gardner JM, et al. Staphylococcus aureus prevention strategies in cardiac surgery: a costeffectiveness analysis. Ann Thorac Surg. 2018;105:47-53.
See Letter page e39.

\section{Commentary: Compliance with the American Association for Thoracic Surgery guidelines will prevent sternal wound infections and minimize postoperative complications in cardiac surgery patients during the COVID-19 pandemic}

Harold L. Lazar, MD

During the current coronavirus disease 2019 (COVID-19) pandemic, patients who have required care in the intensive care unit (ICU) have tended to be older (mean age 66 years) and have a greater incidence of obesity, smoking, and underlying cardiovascular disorders, including diabetes, hypertension, hyperlipidemia, chronic pulmonary disorders, and cardiac disease. ${ }^{1}$ In view of these associated comorbidities, a percentage of these patients will require urgent or emergent cardiac surgery for acute coronary syndromes,

From the Division of Cardiac Surgery, Boston University School of Medicine, Boston, Mass.

Disclosures: Author has nothing to disclose with regard to commercial support. Received for publication April 8, 2020; revisions received April 8, 2020; accepted for publication April 9, 2020; available ahead of print April 13, 2020.

Address for reprints: Harold L. Lazar, MD, 80 East Concord St, Boston, MA 02118 (E-mail: harold.1.lazar@gmail.com).

J Thorac Cardiovasc Surg 2020;160:e44-8

$0022-5223 / \$ 36.00$

Copyright $(2) 2020$ by The American Association for Thoracic Surgery

https://doi.org/10.1016/j.jtcvs.2020.04.016
Check for updates

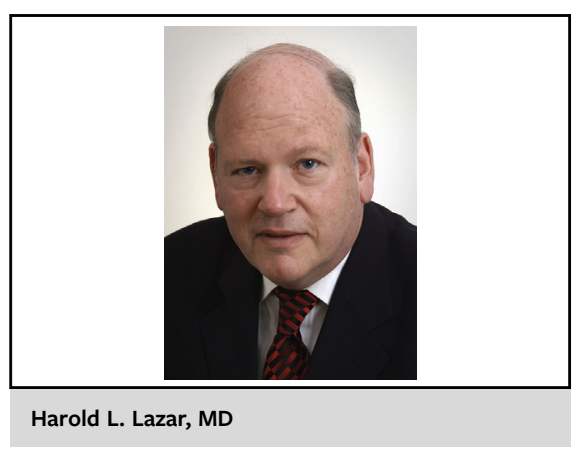

CENTRAL MESSAGE

Compliance with the AATS

guidelines will prevent sternal

wound infections and minimize

postoperative complications in

cardiac surgery patients during

the COVID-19 pandemic.

valvular dysfunction, as well as surgery for aortic dissections and thoracic aneurysms. Unfortunately, the risk factors for mediastinitis following cardiac surgery-obesity, smoking, diabetes, emergent surgery, and hospitalization before surgery-are similar to the profiles of patients who are most likely to require cardiac surgery during the COVID-19 pandemic. $^{2}$ This commentary will review how compliance with the American Association for Thoracic Surgery (AATS) Guidelines for the Prevention of Sternal Wound Infections ${ }^{3}$ will help to minimize infections and wound complications and also help to reduce all postoperative complications in patients requiring cardiac surgery during the COVID-19 pandemic. 